\title{
Ciprofloxacin-loaded Chitosan Nanoparticles as Titanium Coatings: A Valuable Strategy to Prevent Implant- associated Infections
}

Elvira De Giglio ${ }^{1}$, Adriana Trapani ${ }^{2}$, Damiana Cafagna ${ }^{1}$, Concetta Ferretti ${ }^{3}$, Roberta Iatta $^{4}$, Stefania Cometa ${ }^{5}$, Edmondo Ceci ${ }^{6}$, Antonella Romanelli $^{1}$, Monica Mattioli-Belmonte ${ }^{3}$

\author{
${ }^{1}$ Dept. of Chemistry, University of BariAldo Moro,Bari,Italy \\ ${ }^{2}$ Dept. of Pharmacy, University of BariAldo Moro,Bari,Italy \\ ${ }^{3}$ Dept. of Clinical and Molecular Sciences , UniversitàPolitecnicadelle Marche, Ancona, Italy \\ ${ }^{4}$ Dept. of Biomedical Sciences and Human Oncology, University of BariAldo Moro, Bari, Italy \\ ${ }^{5}$ BIOLab, Chemistry \& Industrial Chemistry Department, University of Pisa, Pisa, Italy \\ ${ }^{6}$ Department of Public Health, University of Bari Aldo Moro, Bari, Italy
}

* Corresponding author: elvira.degiglio@uniba.it

Tel/fax.:+39080 5442021/+390805442026

\begin{abstract}
Orthopaedic infections represent one of the major causes of implant failure. Systemic treatment is limited due to dosing, side-effects, patient compliance, treatment length and resistant bacteria. The choice of antibiotic incorporation method has been the subject of many investigations and, nowadays, various vehicles for local drug delivery have been studied. In this work, a novel ciprofloxacin loaded chitosan nanoparticles coating system onto titanium surface has been developed and characterized. The antibiotic release capability of this system and its ability to inhibit the in vitro growth of two of the most common pathogens causing orthopaedic implant-related infections, Staphylococcus aureus and Pseudomonas aeruginosa, have been evaluated. Preliminary biocompatibility data arising from MG63 osteoblast-like cells seeding on the ciprofloxacin-loaded systems have also been discussed. The investigated system represents a promising candidate in view of the development of new antibiotic carriers in situ for preventing titanium implant-associated infections.
\end{abstract}

Keywords: Infection, Titanium, Chitosan Nanoparticles, Ciprofloxacin, Drug Release, Osteoblast

Citation: E.De Giglio et al. Ciprofloxacin-loaded chitosan nanoparticles as titanium coatings: a valuable strategy to prevent implant-associated infections. Nano Biomed. Eng. 2012, 4(4), 163-169.

DOI: $10.5101 /$ nbe.v4i4.p163-169.

\section{Introduction}

Infections in orthopaedic implant surgery are serious complications and a major cause of implant failure [1]. In this respect, a local antimicrobial prophylaxis represents an interesting alternative to systemic administration. For metal implants, their fixation to bone with antibiotic-impregnated cement was reported by Buchholz and Engelbrecht for the first time in 1970 [2]. Today, various vehicles for local antibiotic delivery have been developed: thin biodegradable polymer coatings $[3,4]$; biomimetic hydroxyapatite coatings [5]; modification of titanium implant surfaces by covalently bonded antibiotics [6]. Poor investigation was, indeed, devoted to the employment of hydrogel coatings of metal implants as carrier of antimicrobial agents $[7,8]$. Hydrogel networks, due to their specific characteristic to entrap high water amount, represent an ideal candidate as sustained antibiotic delivery systems. In a previous work we reported the development and characterization of new hydrogel coatings synthesized directly onto titanium substrates by a simple and reproducible electrochemical method $[9,10]$. These coatings resulted able to entrap an antibiotic, exploiting their swelling capabilities. The drug can be then released in situ demonstrating an interesting antibacterial activity [11].

To achieve alternative coatings for titanium implants, biodegradable polymeric nanoparticles (NPs) could be also adopted for antibiotic delivery systems. It has been well documented that nanoparticles based on biopolymers could be employed as vehicles for drug delivery, and in this context, chitosan (CS) is particularly attractive for pharmaceutical applications [12]. Several reasons induce to adopt $\mathrm{CS}$ as a candidate coating layer for titanium implants. Because of CS excellent biocompatibility, biodegradability, nontoxicity and adsorption properties, it has been widely used for drug vehicles also as NPs 
that show high loading and good delivery ability concerning hydrophilic molecules $[13,14]$. On the basis of these findings, in this study chitosan NPs have been investigated to develop a novel colloidal coating on titanium substrate intended as drug delivery system for ciprofloxacin (CIP). This antibiotic has been thoroughly studied in orthopaedic applications and showed in vitro a wide anti-Gram-positive spectrum, especially against $S$. aureus [15] and S. epidermidis [16], the most common pathogens causing orthopaedic implant infections.

In this respect, modern biodegradable CS wound dressings containing CIP have been already proposed for infection treatment [17].

In this work, the formulation and characterization of a chitosan NPs-based coating were described as preliminary steps for the development of potential antibiotic carrier systems in orthopaedic field. The resulting system was characterized in terms of X-ray photoelectron spectroscopy (XPS) analysis of the coating, drug release performances as well as its ability to inhibit the in vitro growth of $S$. aureus and $P$. aeruginosa. Moreover, the effect of this novel drug delivery system on human MG63 osteoblast-like cell viability was also tested.

\section{Experimental Section}

\subsection{Materials}

The following chemicals were obtained from commercial sources and used as received. Chitosan hydrochloride (UP CL 113, Mw $110 \mathrm{kDa}$, deacetylation degree $86 \%$ according to manufacturer instructions) was purchased from Pronova Biopolymer (Norway). Titanium, glycerol (over $99.5 \%$ purity) and ciprofloxacin (MW=331.35 $\mathrm{g} \mathrm{mol}^{-1}$ ) were purchased from Sigma-Aldrich (Italy). Sulfobutyl ether- $\beta$-cyclodextrin sodium salt $\left(\mathrm{SBE}_{7 \mathrm{~m}}-\beta-\mathrm{CD}\right.$, $\mathrm{MW}=2163 \mathrm{Da}$, average substitution degree=6.40) was bought by CyDex, Inc. (USA). Ultrapure water, by Carlo Erba (Italy) was used throughout the study. All other chemicals were reagent grade.

\subsection{Preparation of chitosan nanoparticles-based coatings onto titanium substrates}

Chitosan nanoparticles in the presence and in the absence of CIP were prepared according to a modified ionic gelation method [18]. Briefly, the preparation of an inclusion complex constituted by SBE- $\beta-C D$ and CIP (hereafter called CPX) was carried out for $24 \mathrm{~h}$ at room temperature and under magnetic stirring. The two reagents were poured in double distilled water in equimolar ratio. According to host-guest complexation equilibrium, CIP can exist both in its complexed and free forms inside CPX. In particular, SBE- $\beta-C D$ and CIP concentrations were set at $4.50 \mathrm{mg} \mathrm{mL}^{-1}$ and $0.64 \mathrm{mg} \mathrm{mL}^{-1}$, respectively, in order to provide nanoparticles formation. After $24 \mathrm{~h}$, an aliquot of $0.5 \mathrm{~mL}$ of the $\mathrm{CPX}$ has been used to crosslink $1.5 \mathrm{~mL}$ of an aqueous solution of CS $(0.2 \%, \mathrm{w} / \mathrm{v})$, leading to the formation of chitosan nanoparticles containing CIP (loaded-CSNPs). The $\mathrm{pH}$ of the resulting nanosuspension was equal to 5.2. The volumes above mentioned refer to the maintenance of $\mathrm{CS} / \mathrm{SBE}-\beta-\mathrm{CD}$ mass ratio equal to $4 / 3$ [18]. Unloaded-CSNPs were formulated following the same protocol in the absence of CIP. Freshly prepared suspensions were isolated in Eppendorf tubes with a 10 $\mu \mathrm{L}$ glycerol bed in the bottom of the tube, set to help the resuspension of the formulation. All obtained samples (unloaded-CSNPs and loaded-CSNPs) were centrifuged for $45 \mathrm{~min}$ at $13200 \mathrm{rpm}$. To promote the adsorption of an additional amount of antibiotic on the loaded-CSNPs surface, a further aliquot of CPX was incubated with the final nanoparticles suspension for $3 \mathrm{~h}$ at room temperature without any stirring [14,19]. This procedure allowed us to obtain CPX-enriched-loaded-CSNPs (hereafter called loaded-CSNPs-CPX) that were employed for the antibacterial coatings preparation. The loaded-CSNPsCPX-based coatings were obtained onto titanium sheets depositing by casting on each sample $1.4 \times 10^{-3} \mathrm{mg}$ of loaded-CSNPs which had previously adsorbed $3.5 \times 10^{-2}$ $\mathrm{mL}$ of CPX. Titanium sheets were covered on both sides and after each casting deposition, samples were dried at $40{ }^{\circ} \mathrm{C}$.

\subsection{Physicochemical characterization of nanoparticles}

Particle size and polydispersity index (PI) of all tested NPs were determined in double distilled water by Photon Correlation Spectroscopy (PCS) using a Zetasizer NanoZS (ZEN 3600, Malvern, UK). The determination of the $\zeta$-potential was performed using laser Doppler anemometry with $1 \mathrm{mM} \mathrm{KCl}$ as carrier [20].

\subsection{X-ray photoelectron spectroscopy (XPS) analysis}

XPS has been exploited for the surface characterization of all the investigated nanoparticle systems. XPS spectra were obtained with a Thermo VG Thetaprobe spectrometer (Thermo Fisher Scientific, Inc., Waltham, MA, USA) equipped with a microspot monochromatized Al Kọsource. The Al K $\alpha$ line (1486.6 eV) was used throughout; the base pressure was $10^{-9}$ mbar. Survey scans (binding energy range 0-1200 eV, FAT mode, pass energy $=150 \mathrm{eV}$ ) and detailed spectra (FAT mode, pass energy $=50 \mathrm{eV}$ ) were recorded. Data were analyzed using the Advantage software package, consisting of a non-linear least squares fitting program. Quantification was done by peak area; comparison of data from different elements was enabled by correction with empirically derived atomic sensitivity factors [21]. Data were averaged over at least six samples analyzed. Before XPS analysis, all sample suspensions were deposited by casting onto $\mathrm{Ti}$ substrates.

\subsection{Ciprofloxacin release studies by HPLC}

CIP release studies were perfomed on loadedCSNPs-CPX coatings. The medium for release test was physiological solution $(\mathrm{NaCl} 0.9 \%, \mathrm{w} / \mathrm{v} \%)$ and the assay was carried out up to 5 days in a shaker water bath set at $37{ }^{\circ} \mathrm{C}$ under static conditions. In screw-capped test tubes, each loaded-CSNPs-CPX coated Ti sheet was dipped 
in $2.5 \mathrm{~mL}$ of physiological solution and, at appropriate time intervals, an aliquot $(100 \mu \mathrm{L})$ was withdrawn and analyzed for CIP content by High-performance liquid chromatography (HPLC) as described below. The initial volume of release medium was maintained by refilling $100 \mu \mathrm{L}$ of the same medium after each withdrawal. HPLC analyses of CIP were performed with an Agilent 1260 Infinity (Agilent Technologies, Santa Clara, CA), equipped with a MW detector, a $20 \mu \mathrm{L}$ injection loop, and processed by ChemStation for LC systems Agilent Technologies. For analysis, a reversed phase Synergy (15 cm $\times 4.6 \mathrm{~mm} ; 4 \mu \mathrm{m}$ particles; Phenomenex) column was eluted in isocratic mode [11]. A litre of mobile phase consisted of a mixture of $900 \mathrm{~mL}$ of $50 \mathrm{~mL} \mathrm{~L}^{-1}$ acetic acid, plus $50 \mathrm{~mL}$ acetonitrile and $50 \mathrm{~mL}$ methanol. The flow rate of $1.25 \mathrm{~mL} \mathrm{~min}^{-1}$ was maintained and the column effluent was monitored continuously at 280 nm. Quantification of the compound was carried out by measuring the peak areas in relation to those of standard chromatographed under the same conditions. Standard calibration curves were prepared at $280 \mathrm{~nm}$ wavelength using the eluent above mentioned as solvent and were linear $\left(r^{2}>0.9992\right)$ over the range of tested concentrations (0.1-10 $\left.\mu \mathrm{g} \mathrm{mL}^{-1}\right)$. The retention time of CIP was about 6.0 min. Each experiment was performed in quadruplicate.

\subsection{Coatings antibacterial activity}

The antibacterial activity of loaded-CSNPs-CPX coatings was tested with two different bacterial strains: American Type Culture Collection (ATCC) as reference strains: ATCC 29213 S. aureus and ATCC $27853 P$. aeruginosa. To evaluate the bactericidal activity against the different isolates used, all coatings were previously sterilized by UV radiation for 1 hour/sheet side.

A fresh culture on Mueller Hinton Agar plate was prepared for each isolate. Successively, the bacteria suspension was performed adding 1-2 colonies from the culture into $2 \mathrm{~mL}$ of sterile water in order to have $0.1 \mathrm{Mc}$ Farland turbidity. $1 \mu \mathrm{L}$ of the suspension was inoculated into $14 \mathrm{~mL}$ of Trypticase Soy Broth (TSB) corresponding to about $3 \times 10^{2}$ colony-forming units (CFU) per $\mathrm{mL}$. CSNPs-CPX coatings on Ti sheets were added to the bacteria suspension and incubated at $37^{\circ} \mathrm{C}$ under rotation for $48 \mathrm{~h}$. At each $1 \mathrm{~h}$ interval time, for the first 7 hours $\left(\mathrm{T}_{0}, \mathrm{~T}_{1}, \mathrm{~T}_{2}, \mathrm{~T}_{3}, \mathrm{~T}_{4}, \mathrm{~T}_{5}, \mathrm{~T}_{6}, \mathrm{~T}_{7}\right)$, at $24 \mathrm{~h}\left(\mathrm{~T}_{24}\right)$ and at $48 \mathrm{~h}\left(\mathrm{~T}_{48}\right)$ the total bacterial load (TBL) was determined by plating $1 \mathrm{~mL}$ of the suspension onto a sterile Petri dish and adding Nutrient Agar medium. Each plate was incubated overnight at $37{ }^{\circ} \mathrm{C}$ and the number of colonies $\mathrm{mL}^{-1}$ $\left(\mathrm{CFU} \mathrm{mL}^{-1}\right.$ ) was counted the following day. Unloaded CSNPs coatings on $\mathrm{Ti}$ and bare Ti sheets were tested in the same conditions as controls. For each bacterial strain, several parallel experiments were performed in the same conditions.

\subsection{Biocompatibility assessment}

Cell cultures. MG63 human osteoblast-like cells (ATCC, Rockville, MD, USA) were cultured in a $5 \% \mathrm{CO}_{2}$ controlled atmosphere $\left(\mathrm{T}=37^{\circ} \mathrm{C}\right)$ in Dulbecco's Modified
Eagle's Medium (DMEM, Sigma) supplemented with $10 \%$ fetal bovine serum (FBS), 1\% L-glutamine (Sigma) and $1 \%$ antibiotics (penicillin-streptomycin; GibcoInvitrogen, Milan, Italy). After thawing they were routinely split 1:10 every 2-3 days and used at the fourth passage. The coated Ti specimens were UV sterilized (254 nm) for $2 \mathrm{~h}$ ( 1 hour/sheet side). MG63 cells were detached using $0.25 \%$ trypsin in $1 \mathrm{mM}$ EDTA (Sigma) and plated in triplicate onto the coated Ti sheets in 24well polystyrene tissue culture plates (TCPs) at a density of $1 \times 10^{4}$ cells cm ${ }^{-2}$ for $48 \mathrm{~h}$ at $37^{\circ} \mathrm{C}$.

MTT (3-dimethylthiazol-2,5-diphenyltetrazolium bromide) colorimetric assay. After incubation, the medium was removed; $200 \mu \mathrm{L}$ of MTT (Aldrich 135038, Sigma) solution $\left(5 \mathrm{mg} \mathrm{mL}^{-1}\right.$ in DMEM without phenol red) and $1.8 \mathrm{~mL}$ DMEM were added to all cell monolayers; the multiwell plates were incubated at $37^{\circ} \mathrm{C}$ for a further $4 \mathrm{~h}$. After discarding the supernatants, the dark blue formazan crystals were dissolved by adding $2 \mathrm{~mL}$ of solvent (4\% $\mathrm{HCl} 1 \mathrm{~N}$ in isopropanol absolute) and quantified by spectrophotometry (Secomam, Anthelie light, version 3.8 , Contardi, Italy) at 570 and $690 \mathrm{~nm}$. The results are reported as percentage of bare Ti sheets (controls).

SEM morphological characterization. Specimens were observed with a Philips XL 20 scanning electron microscope (Royal Philips Electronics, Eindhoven, Netherlands) equipped for X-ray microanalysis (EDSPV 9800). Specimens were observed using the secondary electron detector. For SEM analysis, specimens were fixed in $2 \%$ glutaraldehyde in $0.1 \mathrm{M}$ cacodylate buffer ( $\mathrm{pH} 7.4$ ), post-fixed in $1 \%$ osmium tetroxide, dehydrated in increasing ethanol concentrations, critical point dried, mounted on aluminium stubs and gold-sputtered. Data recorded on loaded-CSNPs-CPX coatings were compared with those observed on unloaded-CSNPs coatings and on bare Ti sheets.

\section{Results and Discussion}

\subsection{Physicochemical characterization of nanoparticles}

In this work, a new coating layer based on CIP-loaded chitosan nanoparticles was employed for the first time to obtain an antibiotic delivery system for metal implants aiming at preventing orthopaedic infections.

Currently, several studies focussed their attention on the inclusion of CIP in the cavity of CDs such as the native $\beta-C D$ [22] and the derivative methyl- $\beta-C D$ [23]. Herein, we have selected SBE- $\beta$-CD for the following reasons: (i) it is safe for humans as stated by FDAapproval; (ii) greater water solubility compared with $\beta-C D$ and the derivative methyl- $\beta-C D$ (www.captisol.com); (iii) strong intermolecular linkages between the negative groups present on SBE- $\beta-C D$ and the positively charged amino groups of CS lead to highly compacted NPs [19]. Table 1 shows the physicochemical properties of NPs under investigation.

The use of SBE- $\beta$-CD to crosslink the polysaccharide OenthI 165 
CS led to particle size of $503 \mathrm{~nm}$ and, once CIP is incorporated in the nanosystem, a reduction up to $358 \mathrm{~nm}$ was measured. Moreover, for the two types of particles, a high degree of uniformity of particle size was observed as proved by the low PI values, both for unloaded-CSNPs and loaded-CSNPs nanoparticles. In terms of surface charge, i.e. zeta potential values, both unloaded-CSNPs and loaded-CSNPs exhibited positive values according to the presence of externally located CS chains. These findings confirmed that the modified ionic gelation method used for nanoparticles preparation showed high reproducibility.

Table 1 Physicochemical properties of unloaded-CSNPs and loadedCSNPs.

\begin{tabular}{|l|l|l|l|}
\hline Formulation & Size $(\mathrm{nm})$ & PI & $\zeta(\mathrm{mV})$ \\
\hline Unloaded-CSNPs & $503( \pm 64)$ & $0.23-0.32$ & $+33.8( \pm 0.2)$ \\
\hline Loaded-CSNPs & $358( \pm 48)$ & $0.23-0.35$ & $+34.7( \pm 0.5)$ \\
\hline
\end{tabular}

PI: polydispersity index;

$\zeta$ : zeta potential. Mean \pm S.D are reported, $n=6$.

\subsection{XPS analysis}

XPS has been employed to investigate the surface chemical composition of unloaded-CSNPs, loadedCSNPs and loaded-CSNPs-CPX coatings onto Ti sheets. In Fig. 1 the survey scans of unloaded-CSNPs, loadedCSNPs and loaded-CSNPs-CPX coatings were compared. The spectrum of unloaded-CSNPs showed the typical nanoparticles components (carbon, oxygen, nitrogen and sulphur), as expected (see Table 2). The presence of sulphur was due to the use of SBE- $\beta$-CD as cross-linking agent.

It was noted that the introduction of the antibiotic in the nanoparticles did not only add contributions to carbon, oxygen and nitrogen spectral regions, but also introduced the characteristic fluorine signal to the XPS spectra. Therefore, fluorine and sulphur can be considered

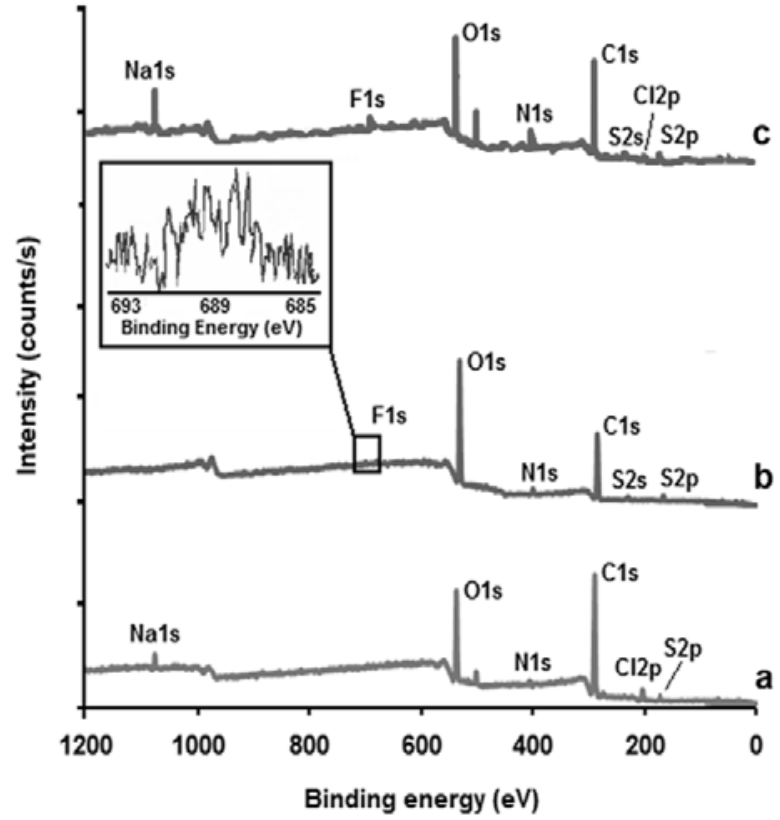

Fig. 1 Comparison of survey spectra relevant to unloaded-CSNPs (a), loaded-CSNPs (b) and loaded-CSNPs-CPX (c) coatings.
Table 2 Surface composition (atomic percentages: At $\%$ ) of unloadedCSNPs, loaded-CSNPs and loaded-CSNPs-CPX coatings onto Ti sheets determined by XPS.

\begin{tabular}{|c|c|c|c|}
\hline Element & \multicolumn{3}{|c|}{ At\% } \\
\hline & $\begin{array}{c}\text { Unloaded- } \\
\text { CSNPs }\end{array}$ & Loaded-CSNPs & $\begin{array}{c}\text { Loaded-CSNPs- } \\
\text { CPX }\end{array}$ \\
\hline $\mathrm{C}$ & 72.1 & 62.8 & 64.8 \\
\hline $\mathrm{O}$ & 18.1 & 30.5 & 24.9 \\
\hline $\mathrm{N}$ & 2.6 & 3.0 & 3.5 \\
\hline $\mathrm{S}$ & 1.8 & 2.8 & 3.8 \\
\hline $\mathrm{F}$ & - & 0.2 & 0.7 \\
\hline $\mathrm{Cl}$ & 4.3 & 0.2 & 1.1 \\
\hline $\mathrm{Na}$ & 1.2 & 0.1 & 1.7 \\
\hline
\end{tabular}

XPS marker elements for antibiotic and SBE- $\beta-C D$, respectively.

The atomic percentages of fluorine were equal to $0.2 \%$ for CSNPs and $0.7 \%$ for CSNPs-CPX, whereas the area ratios $\mathrm{F} 1 \mathrm{~s} / \mathrm{S} 2 \mathrm{p}$, recorded on loaded CSNPs and loadedCSNPs-CPX, were equal to 0.07 and 0.18 respectively. These findings suggested a predominant presence of the antibiotic on the loaded-CSNPs-CPX thanks to the additional amount of the complex adsorbed onto loadedCSNPs after their preparation. In particular, the higher F1s/S2p area ratio value for loaded-CSNPs-CPX system let us to argue that some antibiotic (in its cationic form due to the $\mathrm{pH}$ value of the medium=5.2) should interact electrostatically with the anionic bearing groups of SBE$\beta-\mathrm{CD}$ rather than via the host-guest inclusion complex. Indeed, such interaction could be reasonably understood taking into account that the host SBE- $\beta-C D$ is a hydrophilic cyclodextrin where the $-\mathrm{SO}_{3}{ }^{-}$functionalities are exposed outside the cavity. On the other hand, a lipophilic cyclodextrin such as methyl- $\beta-C D$ was already seen to interact with CIP, using the piperazynil ring encapsulated inside the cavity [23].

Moreover, the spectra relevant to the three investigated coatings evidenced the presence of signals of chlorine and sodium, relevant to chitosan and SBE- $\beta-C D$, respectively.

The XPS investigation allowed us to point out the difference between the unloaded-CSNPs and CIPmodified coatings (loaded-CSNPs and loaded-CSNPsCPX) in terms of surface features. Altogether, XPS analyses suggested only a slight presence of CIP on the loaded-CSNPs surface although its existence within NPs matrix cannot be excluded. The external location of CIP could provide a first prompt biological availability in the site of implantation. In the future, further analytical investigations will be performed by XPS analyses, through etching procedures, to verify the presence and the distribution of the antibiotic inside the core of the loadedCSNPs system.

\subsection{Ciprofloxacin release from loaded-CSNPs-CPX coat-ings on titanium substrates}

To verify the antibiotic release performances of the investigated coatings, CIP release studies have been 


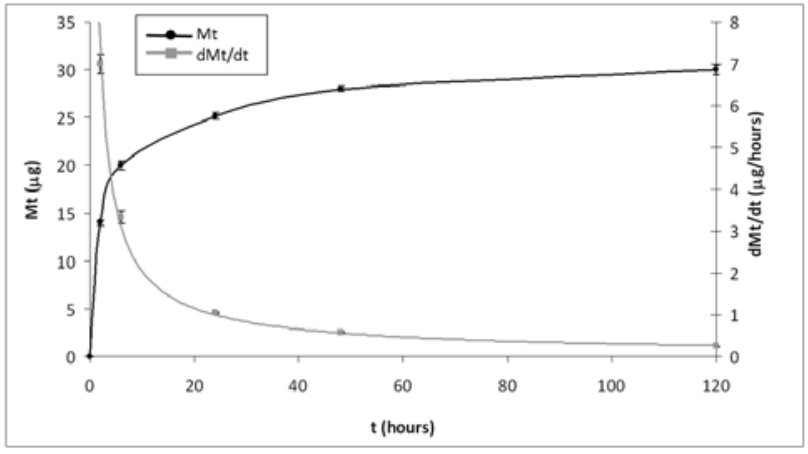

Fig. 2 Ciprofloxacin amount released over time (black curve) and ciprofloxacin release rate (grey curve) from loaded-CSNPs-CPX coatings.

carried out by HPLC . In Fig. 2 the antibiotic release rate and the antibiotic amount released over time from loadedCSNPs-CPX coatings were reported. From the release rate profile, it can be observed that CIP was released from the nanoparticle system with an initial rapid release over the first 6-7 h (approximately the 60\% of the total release) and then a slow release from the nanoparticles was displayed. In this case, it seems that the zero order and Higuchi's models failed to explain CIP release mechanism due to swelling (upon hydration) or to a possible erosion of the chitosan [24] matrix.

The burst effect observed in the curve of the antibiotic amount released over time was already studied by Chandran et al. [22] which reported the complex $\beta$-cyclodextrin-CIP incorporated in the CS matrix of an Ocusert device. The burst effect could also be related to the amount of CIP externally located as evidenced by XPS studies and, from a clinical viewpoint, it could be encouraging in terms of a prompt supply of the same antibiotic. Moreover, in agreement with Blanchemain et al. [23] the observed release profile could be due to the ionic strength of physiological solution, which exerted a "salting out effect", inducing the desorption of CIP adsorbed onto the support. As shown in the curve of the antibiotic amount released over time, the highest level of $\mathrm{CIP}$ delivered was equal to $30 \mu \mathrm{g}$ after $120 \mathrm{~h}$. Taking into account the loaded-CSNPs-CPX preparation procedure, the $77 \%$ of the total amount released raised from the adsorbed CPX, whereas the complementary moiety can be ascribed to the reservoir system constituted by loadedCSNPs. Notably, a CIP concentration equal to $12 \mu \mathrm{g}$ $\mathrm{mL}^{-1}$ after $120 \mathrm{~h}$ was higher than the MIC values of the main pathogens involved in the orthopaedic infections. On the other hand, the CIP release profile from loadedCSNPs did not provide any burst effect showing a quite slow trend with $2.8 \mu \mathrm{g} \mathrm{mL} \mathrm{m}^{-1}$ of CIP delivered after 120 h. Therefore, in the following studies, we focussed our attention on loaded-CSNPs-CPX system since the localization of CIP both internally and externally can enhance the coating antibacterial performances.

\section{Antibacterial activity of loaded-CSNPs-CPX coatings}

Whenever an implant device is placed in vivo, bacteria can become adherent to either the metallic or the polymeric component and will compete with host cells for colonization of the implant surface. The most common pathogens causing orthopaedic implant infections are S.aureus, S.epidermidis and P. aeruginosa, which are responsible for up to $60 \%$ of all prosthetic hip implant infections $[15,16]$. In this work, a novel CIPloaded chitosan nanoparticle coating has been employed to release the antibiotic from the metal devices surface directly in the implant site. In detail, the antibacterial activity of loaded-CSNPs-CPX coatings was verified against two of the main microorganisms responsible of the orthopaedic infections, S. aureus (Gram+) and $P$. aeruginosa (Gram-).

As controls, two different samples were used: bare Ti sheets and unloaded-CSNPs coatings onto Ti sheets. Both control samples did not influenced the microbial proliferation of $P$. aeruginosa and $S$. aureus, therefore, in the following microbiological tests only unloadedCSNPs coatings were used as positive controls. The Fig. 3 showed that the novel drug delivery system was able to inhibit $S$. aureus and $P$. aeruginosa growths from the first hours of incubation. On the contrary, the employment of the loaded-CSNPs system did not produce any relevant antibacterial effect (data not shown). These findings are in good agreement with the in vitro release of CIP from NPs, suggesting that the delivery of the antibiotic via the burst effect mechanism allows to reach an excellent antibacterial activity.
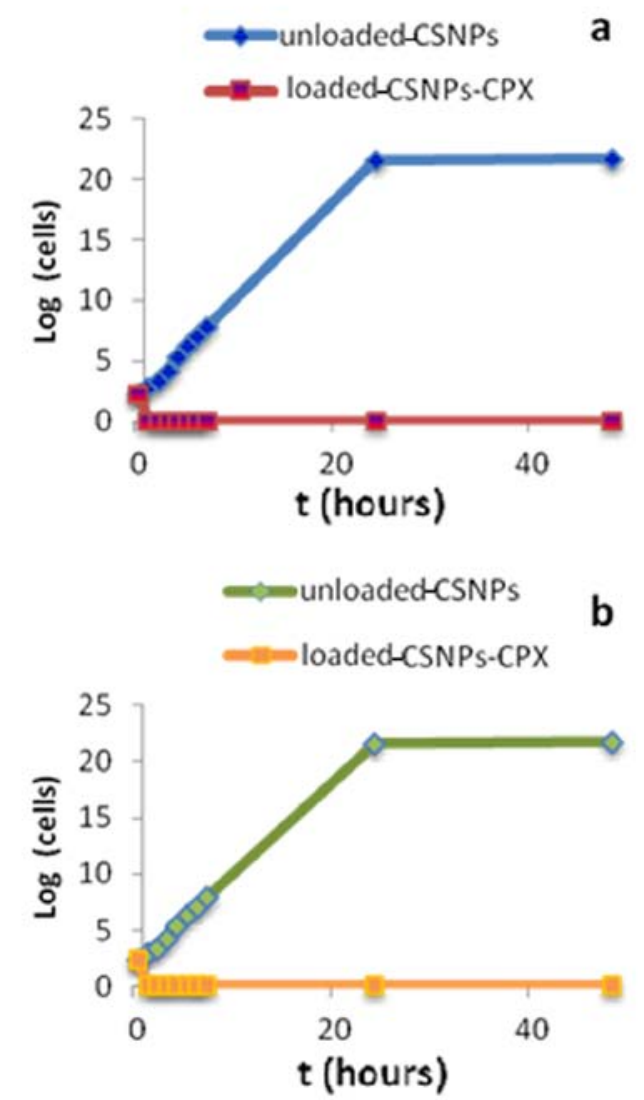

Fig. $3 S$. aureus (a) and P. aeruginosa (b) growth at different time points in presence of loaded-CSNPs-CPX and unloaded CSNPs (control) coatings on titanium sheets 


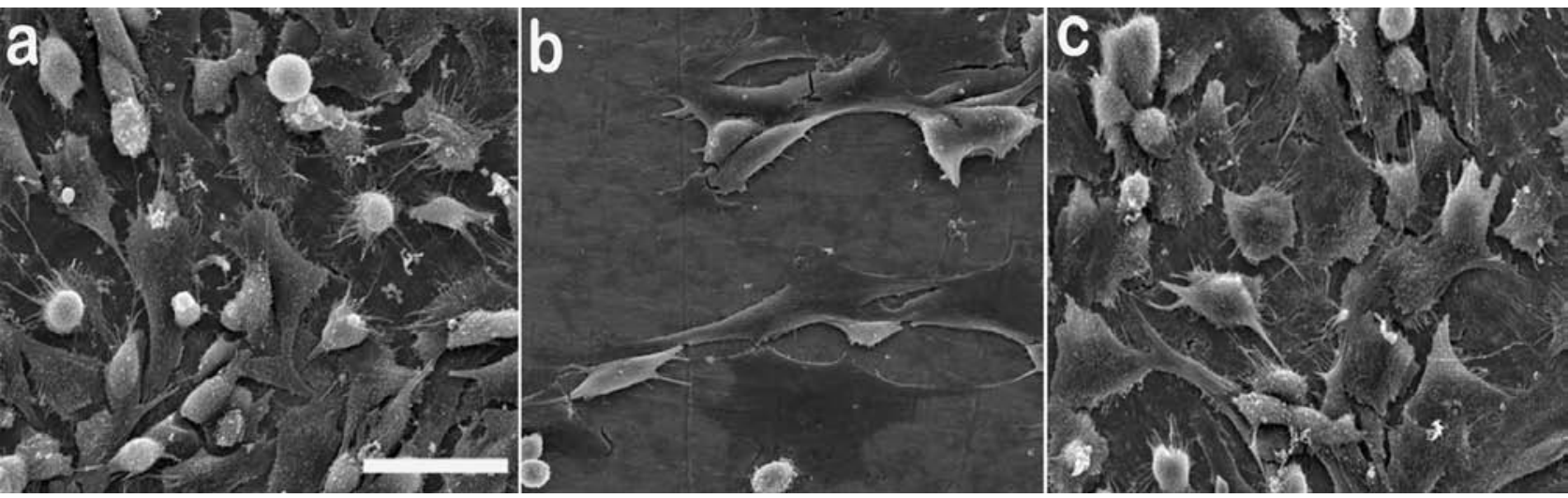

Fig. 4 SEM micrographs of MG-63 cells cultured for $48 \mathrm{~h}$ on bare Ti sheets (a), unloaded- CSNPs coatings (b) and loaded-CSNPsCPX coatings onto Ti (c). Scale bar $=50 \mu \mathrm{m}$.

\subsection{Biocompatibility investigations}

The biocompatibility of the loaded-CSNPs-CPX coatings onto Ti substrates is strictly related to cell behaviour when cells are in contact with this novel device. Adhesion and spreading of osteoblasts to a biomaterial are the first step of cell-material interaction, and the quality of this process will influence the subsequent cell proliferation and/or differentiation. MG63 human osteoblast-like cells have often been used as a model to investigate osteoblast behaviour on polymer surfaces $[25,26]$.

MTT tests and SEM observations were carried out with MG63 human osteoblast-like cells, with the aim to evaluate the response of bone cells to this new loadedCSNPs-CPX coating. Since the biocompatibility of chitosan-based material used for the preparation of the nanoparticles is widely reported in the literature [27], the first compatibility test has been performed to evaluate the possible correlation between concentration-toxicity of the other compounds used for the formulation of the CSNPs. In this respect, MTT viability test on MG63 human osteoblast-like cells was carried out adding to the culture medium pure CIP, SBE- $\beta$-CD and the complex $\mathrm{CPX}$ at different concentrations (data not shown). Results underlined that a CPX concentration equal to $0.123 \mathrm{mg}$ $\mathrm{mL}^{-1}$ was toxic for the osteoblasts (MTT values lower than $20 \%$ vs TCPs as control), while an amount of the complex ten times lower $\left(0.0123 \mathrm{mg} \mathrm{mL}^{-1}\right)$, almost equal to that used to obtain the coatings investigated in this work, was not toxic for the cells (MTT values comparable to TCPs control). The MTT test, performed on MG63 human osteoblast-like cells seeded onto the nanoparticlescoated Ti substrates evidenced a different viability in cells seeded onto unloaded-CSNPs coatings in comparison with MG63 cultured on loaded-CSNPs-CPX coatings (61 $\pm 6 \%$ vs $78 \pm 3 \%$; $<<0.05$ ).

SEM morphological observations (Fig. 4) performed on cells seeded on loaded-CSNPs-CPX coatings and on two types of controls (unloaded-CSNPs coatings and bare Ti sheets) suggested that the lower MTT value detected at $48 \mathrm{~h}$ on unloaded-CSNPs was probably related to a lower cell adhesion and not to a direct cytotoxicity. Our findings are related to a preliminary biocompatibility evaluation of the proposed coating performed $48 \mathrm{~h}$ after seeding. We, therefore, hypothesize that this result may be correlated to the different surface chemistry of unloaded-CSNPs and loaded-CSNPs-CPX coatings. In particular, it can be argued that a predominance of SBE- $\beta-C D$ sulfonated groups on loaded-CSNPs-CPX could play a crucial role in osteoblast-like cell adhesion as previously observed by other authors. Indeed, Chaterji et al. demonstrated that sulfonated matrices enhanced the adhesion and proliferation of osteoblast-like cells by simulating the components of the natural osteogenic extracellular matrix [28]. Finally, the excellent increase of MG63 cell adhesion evidenced on the loaded-CSNPs-CPX system was not detected in the case of the loaded-CSNPs one, that displayed after $48 \mathrm{~h}$ a cell adhesion behaviour similar to the one from unloaded-CSNPs.

Overall, these preliminary biocompatibility data are encouraging. However, it will be necessary to further investigate if the proposed coating does not affect osteoblasts proliferation and differentiation.

\section{Conclusion}

In the present work, a new antibiotic-loaded nanoparticle system was proposed as bioactive coating for titanium implants. The nano-coatings exhibited small diameters and narrow size distribution and showed relatively high ciprofloxacin contents. XPS analysis provided information about the surface location of the antibiotic onto the nanoparticle system; from HPLC the amount of CIP released by the coating was estimated. The antibacterial efficacy of this local antibiotic delivery system was evaluated by in vitro test with $S$. aureus and $P$. aeruginosa. Preliminary coating biocompatibility was also investigated using MG63 osteoblast-like cells. Finally, this new nanoparticle-based coating can be considered an interesting nontoxic, biocompatible and freely available drug delivery system which could be used prophylactically for the reduction of bacterial infections frequently related to orthopaedic surgery. Further biocompatibility studies are currently in progress in order to assess if this system could affect osteoblast function in bone remodelling. 


\section{Acknowledgements}

This project was financed by Università degli Studi di Bari Aldo Moro (FAR to E. De Giglio) and FIRB grant project RBAP10MLK7 (Università Politecnica delle Marche).We acknowledge Professor G. Trapani of Università degli Studi di Bari Aldo Moro for useful discussions about nanoparticles design.

\section{References}

1 Qiu Y., Zhang N., An Y.H., Wen X., Biomaterial strategies to reduce implant-associated infections, Int. J. Artif. Organs 2007; 30: 828-841.

2 Buchholz H.W., Engelbrecht H., Uber die depotwir-kung einiger antibiotica bei vermischung mit dem kunstharz Palacos, Chirurg. 1970; 41:51.

3 Lucke M., Schmidmaier G., Sadoni S., Wildemann B., Schiller R., Haas N.P., Raschkea M., Gentamicin coating of metallic implants reduces implant-related osteomyelitis in rats, Bone. 2003; 32: 521-531.

4 Hongshuai L., Ogle H., Bingbing J., Hagar M., Bingyun L., Cefazolin embedded biodegradable polypeptide nanofilms promising for infection prevention: a preliminary study on cell response, J. Orthop. Res. 2010; 28: 979-1118.

5 Brohede U., Forsgren J., Roos S., Mihranyan A., Engqvist H., Strømme M., Multifunctional implant coatings providing possibilities for fast antibiotics loading with subsequent slow release, J. Mater.Sci. Mater. Med. 2009; 20: 1859-1867.

6 Binoy J., Antoci V., Zeiger A.R., Wickstroml E., Hickok N.J., Vancomycin covalently bonded to titanium bead kills Staphylococcus aureus, Chem. Biol. 2005; 9:1041-1048.

7 De Giglio E., Cafagna D., Cometa S., Allegretta A., Pedico A., Giannossa L.C., Sabbatini L., Mattioli-Belmonte M., Iatta R., An innovative, easily fabricated, silver nanoparticle-based titanium implant coating: development and analytical characterization, Anal. Bioanal. Chem. 2012; in press.

8 Giammona G., Pitarresi G., Palumbo F., Romano C.L., Meani E., Cremascoli E., Antibacterial hydrogel and use thereof in orthopedics. Patent Cooperation Treaty Application, 2010.

9 De Giglio E., Cometa S., Satriano C., Sabbatini L., Zambonin P.G., Electrosynthesis of hydrogel films on metal substrates for the development of coatings with tunable drug delivery performances. $J$. Biomed. Mater. Res.A 2009;88:1048-1057.

10 De Giglio E., Cometa S., Calvano C.D., Sabbatini L., Zambonin P.G., Colucci S., Benedetto A.D., Colaianni G., A new titanium biofunctionalized interface based on poly(pyrrole-3-acetic acid) coating: Proliferation of osteoblast-like cells and future perspectives, J. Mater. Sci. Mater. Med. 2007; 18: 1781-1789.

11 De Giglio E., Cometa S., Ricci M.A., Cafagna D., Savino A.M., Sabbatini L., Orciani M., Ceci E., Novello L., Tantillo G.M., MattioliBelmonte M., Ciprofloxacin-modified electrosynthetized hydrogel coatings to prevent titanium-implant-associated infections, Acta. Biomater. 2011; 7: 882-891.

12 Agnihotri S.A., Mallikarjuna N.N., Aminabhavi T.M., Recent advances on chitosan based micro and nanoparticles in drug delivery, J. Control. Release. 2004; 100: 5-28.

13 Park J.H., Saravanakumar G., Kim K., Kwon I.C., Targeted delivery of low molecular drugs using chitosan and its derivatives, Adv. Drug Deliv. Rev. 2010; 62:28.
14 De Giglio E., Trapani A., Cafagna D., Sabbatini L., Cometa S., Dopamine-loaded chitosan nanoparticles: formulation and analytical characterization. Anal, Bioanal, Chem. 2011; 400: 1997-2002.

15 Mulligan M.E., Murray-Leisure K.A., Ribner B.S., Methicillin resistant s. aureus: a consensus review of the microbiology pathogenesis, and epidemiology with implications for prevention and management, Am. J. Med. 1993; 94:313.

16 Gagnon R.F., Richards G.K., Subang R., Experimental $s$. epidermidis implant infection in the mouse. Kinetics of rifampin and vancomycin action, ASAIO J. 1992; 38(3):596.

17 Radhakumary C., Molly A., Sreenivasan, K., Drug loaded thermoresponsive and cytocompatible chitosan based hydrogel as a potential wound dressing, Carbohyd Polym. 2011; 83: 705-713.

18 Trapani A., Lopedota A., Franco M., Cioffi N., Ieva E., GarciaFuentes M, Alonso MJ. A comparative study of chitosan and chitosan/cyclodextrin nanoparticles as potential carriers for oral delivery of small peptides, Eur. J. Pharm. Biopharm. 2010; 75: 26-32.

19 Hirsjärvi S., Qiao Y., Royere A., Bibette J., Benoit J.P., Layer-by-layer surface modification of lipid nanocapsules. Eur. J. Pharm. Biopharm. 2010; 76: 200-207.

20 Montenegro L., Trapani A., Latrofa A., Puglisi G., In vitro evaluation on a model of Blood Brain Barrier of Idebenone loaded solid lipid nanoparticles. J. Nanosci. Nanotechno. 2012; 12: 330-337.

21 Cometa S., Chiellini F., Bartolozzi I., Chiellini E., De Giglio E., Sabbatini L., Surface segregation assessment in poly( $\varepsilon$-caprolactone)poly(ethylene glycol) multiblock copolymer films, Macromol. Biosci. 2010; 10: 317-327.

22 Chandran S., Shirwaikar C.A., Sarala A., Devi Kiron S.S., Development and evaluation of chitosan ocuserts containing ciprofloxacin and b-CD complex, Int. J. Pharm. Tech. Res. 2010; 246-252.

23 Blanchemain N., Karrout Y., Tabary N., Neut C., Bria M., Siepmann J., Hildebrand H.F., Martel B., Methyl-b-cyclodextrin modified vascular prosthesis: Influence of the modification level on the drug delivery properties in different media. Acta. Biomater. 2011; 7:304-314.

24 Peppas N.A., Analysis of fickian and non-fickian drug release from polymers, Int. J. Pharm. 1985; 15:25-35.

25 Rausch-Fanm X., Qu Z., Wieland M., Matejka M., Schedle A., Differentiation and cytokine synthesis of human alveolar osteoblasts compared to osteoblast-like cells (MG-63) in response to titanium surfaces, Dental. Mater. 2008; 24:102-110.

26 Mattioli-Belmonte M., Orciani M., Ferretti C., Orsini G., De Giglio E., Di Primio R., Cell behaviour on bioactive polymeric coatings, Ital. J. Anat. Embryol. 2010; 115(1/2): 127-133.

27 Martino A.D., Sittinger M., Risbud M.V., Chitosan: a versatile biopolymer for orthopedic tissue-engineering, Biomaterials. 2005; 26: 5983-5990.

28 Chaterji S., Gemeinhart R.A., Enhanced osteoblast-like cell adhesion and proliferation using sulfonate-bearing polymeric Scaffolds. J. Biomed. Mater Res. A 2007; 83: 990-998.

Copyright:(c) 2012 E.De Giglio et al. This is an open-access article distributed under the terms of the Creative Commons Attribution License, which permits unrestricted use, distribution, and reproduction in any medium, provided the original author and source are credited. 